\title{
Nematic Quantum Hall Fluid Without Stripes
}

\author{
Pomeranchuk instability of composite Fermi liquid \\ Authors: K. Lee, J. Shao, E-A Kim, F.D.M. Haldane, and E. H. Rezai \\ arXiv:1802.08261

\begin{abstract}
Recommended with a Commentary by Steven A. Kivelson, Stanford University, Stanford, CA 94305
\end{abstract}

The two-dimensional electron gas (2DEG) in a transverse magnetic field is about the simplest experimentally realizable highly correlated electron fluid, and has thus played a central role in the subject generally. In addition to having been the foundational system in which topological order - as reflected in various quantum Hall liquid states - was first explored, it has also proven to be an ideal laboratory for the investigation of the emergence of various forms of spontaneously broken symmetries. In particular, it has been observed under a wide variety of circumstances $[2,3,4,5,6,7,9,10]$ - perhaps universally in conditions in which the chemical potential lies in a partially filled higher Landau level - that states form that spontaneously break the rotational symmetry down to a residual $C_{2}$.

An important conceptual issue is that it is unclear when this is the full extent of the symmetry breaking - i.e. if the phase giving rise to the macroscopically observed anisotropy is a nematic state - or if this anisotropy arises as a component of a more complex pattern of translation and rotational symmetry breaking, e.g. some form of stripe ordered density wave phase. Often, there is no direct evidence of any form of density wave order in the experimental observations. None-the-less, it is frequently assumed that such density-wave order exists to the extent that any system exhibiting such anisotropies is often described in the literature as a "stripe phase." One reason for this is that it is straightforward to visualize how rotational symmetry breaking can arise from stripe order, and indeed in many cases it is possible to write a Hartree-Fock wave-function for a stripe phase, while a purely nematic phase resists characterization in such a classical sense.

Still, there are at least two ways one can get a theoretical handle on a purely nematic electron fluid in this problem: i) One is to imagine a melted stripe phase - a "nematic close to a smectic" in the language of classical liquid crystals.[11] Such a description is natural[12] at non-zero temperature, where the proliferation of dislocations inevitably destroys stripe order, but can leave (quasi-long-range) orientational order intact. It is, however, much more difficult to treat the problem of the partial quantum melting of a stripe phase to obtain an understanding of a $\mathrm{T}=0$ nematic Fermi fluid. ii) The other approach is to start from a Fermi liquid in which a $l=2$ Pomeranchuck instability leads to an elliptical distortion of the Fermi surface. In the quantum Hall context, especially in conditions of a nearly half-filled Landau level, such a nematic Fermi fluid presumably involves a Pomeranchuk instability of 
a composite Fermi liquid. Such a state is easy to visualize intuitively,[13] but extremely difficult to handle in any quantitative fashion. For this reason, it has remained unclear whether such a state is a physically plausible candidate to account for the anisotropic states observed in experiment.

In this context, the recent paper of Lee et al represents not just a notable calculational advance, [14] but also a conceptually important contribution to the field. The explicit calculations exploit advances that have been made in writing composite Fermion wave-functions projected onto a single Landau level. Variational energies can then be numerically computed for a large enough number of particles that finite size effects can plausibly be neglected. No attempt was made to construct an actual nematic state - rather, energies of various small deformations of the isotropic composite Fermi liquid state were computed from which the values of the various Fermi liquid parameters, $F_{l}$, could be extracted. The pseudo-potentials used in the variational calculation not only depend on the assumed Landau level index, $\mathrm{n}$, in the usual way, but on the short-distance behavior of the Coulomb interaction as well. This the authors characterize by introducing a dimensionless parameter, $\eta$, which loosely can be thought of as the thickness of the 2DEG in units of the magnetic length.

Carrying the usual considerations of Fermi liquid theory over to the composite Fermi liquid, the isotropic state is assumed to be stable so long as $F_{l}>-1$ for all $l>1$, while a Pomerancuk instability occurs whenever $F_{l} \leq-1$. (In general, and in the composite Fermi liquid in particular, special considerations apply to $F_{0}$ and $F_{1}$.) Strikingly, the variational calculations show that $F_{2}$ is a strongly decreasing function of Landau level index (at least for $0 \leq n \leq 2$ ) and of decreasing $\eta$. In particular, for $n=2, F_{2}$ computed in this way is less than -1 for small enough $\eta$, implying the existence of a nematic instability. No other angular momentum channel comes close.

While compelling arguments[16, 17] can be made that stripe phases are likely the true ground-states for half-filled Landau levels with large enough Landau index, and while it seems likely that an isotropic composite Fermi liquid of one sort or another is typically the ground-state for $n=0$, the present results lend considerable weight to the proposition that under many circumstances (e.g. for small enough $\eta$ ) and for intermediate $\mathrm{n}$, the ground state is a nematic composite Fermi fluid.

\section{References}

[1] Pomeranchuk instability of composite Fermi liquid, K. Lee, J. Shao, E-A Kim, F.D.M. Haldane, and E. H. Rezai, arXiv:1802.08261.

[2] Evidence for an Anisotropic State of Two-Dimensional Electrons in High Landau Levels, M. P. Lilly, K. B. Cooper, J. P. Eisenstein, L. N. Pfeiffer, and K. W. West, Phys. Rev. Lett. 82, 394 (1999).

[3] Strongly Anisotropic Transport in Higher Two-Dimensional Landau Levels, R. R. Du, D. C. Tsui, H. L. Stormer, L. N. Pfeiffer, K. W. Baldwin, and K. W. West, Solid State Commun. 109, 389 (1999). 
[4] Competing Quantum Phases in the Second Landau Level in the Low Density Limit, W. Pan, A. Serafin, J. S. Xia, L. Yin, N. S. Sullivan, K. W. Baldwin, K. W. West, L. N. Pfeiffer, and D. C. Tsui, Phys. Rev. B 89, 241302 (2014).

[5] Observation of a transition from a topologically ordered to a spontaneously broken symmetry phase, N. Samkharadze, K. A. Schreiber, G. C. Gardner, M. J. Manfra, E. Fradkin, and G. A. Csathy, Nat. Phys. 12, 191 (2016).

[6] Electron-Electron Interactions and the Paired-to-Nematic Quantum Phase Transition in the Second Landau Level, K. A. Schreiber, N. Samkharadze, G. C. Gardner, Y. LyandaGeller, M. J. Manfra, L. N. Pfeiffer, K. W. West and G. A. Csathy, Nature Communications 9, 2400 (2018).

[7] Tilt-Induced Anisotropic to Isotropic Phase Transition at $\nu=5 / 2$, Xia, Jing; Cvicek, Vaclav; Eisenstein, J. P.; et al., Phys. Rev. Lett. 105, 176807 (2010).

[8] Possible nematic to smectic phase transition in a two-dimensional electron gas at halffilling, Q. Qian, J. Nakamura, S. Fallahi, G. C. Gardner, M. Manfra, Nature Communications, 8, 1536 (2017).

[9] Evidence for $\nu=5 / 2$ fractional quantum Hall nematic state in parallel magnetic fields, Y. Liu, S. Hasdemir, M. Shayegan, L. N. Pfeiffer, K. W. West, and K. W. Baldwin, Phys. Rev. B 88, 035307 (2013).

[10] Strong Transport Anisotropy in Ge/SiGe Quantum Wells in Tilted Magnetic Fields, Q. Shi, M. A. Zudov, C. Morrison, et al, Phys. Rev. B 91, 201301 (2015).

[11] P. M. Chaikin and T. C. Lubensky, Principles of Condensed Matter Physics, (Cambridge University Press, 2012)

[12] E. Fradkin and S. A. Kivelson, Phys. Rev. B 59, 8065 (1999).

[13] Quantum Theory of a Nematic Fermi Fluid, V. Oganesyan, S. Kivelson, and E. Fradkin, Phys. Rev. B 64, 195109 (2001).

[14] In agreement with the present results, a previous variational Monte Carlo study of Doan and Manousakis[15] concluded that a nematic composite Fermi liquid was likely the ground-state phase of a half-filled Landau level when the thickness parameter, $\eta$, is sufficiently small. However, in this calculation the projection onto a single Landau level was treated only approximately, leaving some ambiguity in the result.

[15] Q. M. Doan and E. Manousakis, Phys. Rev. B 75, 195433 (2007).

[16] A. A. Koulakov, M. M. Fogler, and B. I. Shklovskii, Phys. Rev. B 76, 499 (1996).

[17] R. Moessner and J. T. Chalker, Phys. Rev. B 54, 5006 (1996). 